\title{
Infrared spectra of dimethylnaphthalenes in the gas phase
}

\author{
Prasanta Das, E. Arunan*, Puspendu K. Das ** \\ Department of Inorganic and Physical Chemistry, Indian Institute of Science, Bangalore 560012, India \\ Received 1 October 2007; received in revised form 7 January 2008; accepted 15 January 2008 \\ Available online 21 January 2008
}

\begin{abstract}
We report the infrared spectra at $0.5 \mathrm{~cm}^{-1}$ resolution of three dimethylnaphthalenes (DMNs), namely 1,5-DMN, 1,6-DMN and 2,6-DMN in the gas phase at an elevated temperature recorded with the help of a variable path-length cell. DFT calculations have been carried out at B3LYP levels of theory with $6-31 \mathrm{G}^{*}$ as basis set to determine the harmonic frequencies and intensities of the DMNs to assign the experimentally observed spectra. We have compared the experimentally observed and theoretically calculated spectra of the dimethylnaphthalenes and assignments have been made. The observed and predicted frequencies and relative intensities are generally in good agreement. The intense aromatic $\mathrm{C}-\mathrm{H}$ out-ofplane bending vibration observed around $800 \mathrm{~cm}^{-1}$ and three methyl $\mathrm{C}-\mathrm{H}$ symmetric and asymmetric stretching vibrations around $2900 \mathrm{~cm}$ have been recognized as unique bands to identify various DMNs in a mixture. The high-resolution IR spectroscopy of these three important polycyclic aromatic hydrocarbons which are present in the atmosphere have been discussed.
\end{abstract}

(C) 2008 Elsevier B.V. All rights reserved.

Keywords: Polycyclic aromatic hydrocarbons; Dimethylnaphthalenes; Infrared spectra; Harmonic analysis; DFT calculation

\section{Introduction}

Naphthalene and alkylated naphthalenes are semi-volatile polycyclic aromatic hydrocarbons (PAHs) present in the atmosphere mostly in the gas phase [1]. Diesel fuel contains alkyl-PAHs including methyl naphthalenes (MNs) and dimethylnaphthalenes (DMNs) [2,3]. In urban areas emission from diesel-fueled vehicles puts these compounds [4,5] in the atmosphere. The alkylated PAHs have also been identified in individual interplanetary dust particles (IDPs) in as early as 1993 [6].

The toxicity, carcinogenicity and mutagenicity of DMNs are different, and thus, it is important to identify and distinguish them at low concentrations. Gas chromatography/Fourier transform infrared studies of gas phase PAHs and their methylated derivatives have been reported [7], where infrared spectra of 33 PAHs including those of 2-methylanthracene, 9methylanthracene, 1-methylphenanthrene, 9,10-dimethylanthracene and 7,12-dimethylphenanthrene have been included.

\footnotetext{
* Corresponding author. Tel.: +91 8022932662 ; fax: +91 8023601552.

** Corresponding author. Tel.: +91 80 22932828; fax: +91 8023601552.

E-mail addresses: arunan@ipc.iisc.ernet.in (E. Arunan), pkdas@ipc.iisc.ernet.in, pkdasipc@gmail.com (P.K. Das).
}

Theoretical infrared spectra of PAHs on nitrogen substitution, oxygen substitution [8a,b] and methyl substitution [9] have also been reported. The low-resolution gas phase infrared spectra of 1,5- and 2,6-DMNs have been reported in the NIST chemistry web book [10], but vibrational assignments have not been done.

We have studied the FT-IR spectra of 1,5-, 1,6- and 2,6DMNs in the gas phase at low pressure with the help of a long variable path-length cell. The experimental spectra are compared with theoretically calculated spectra at B3LYP/6$31 \mathrm{G}^{*}$ level of theory. The motivation to do this work is to identify and distinguish DMNs by IR spectroscopy.

\section{Materials and methods}

DMNs used in this investigation are 1,6-dimethylnaphthalene (98\%, Fluka), 1,5-dimethylnaphthalene (98\%, Aldrich Chem. Co.) and 2,6-dimethylnaphthalene (99.8\%, Oekanal) which were obtained commercially.

FT-IR spectra of the compounds were recorded in a Thermonicolet (Nexus 870) spectrometer. PAHs, in general, have insufficient vapour pressure at room temperature and thus it is difficult to get PAHs in the gas phase from their solid or liquid phase. Kurtz used a stainless steel cell to get the IR spectrum of coronene at elevated temperatures [11]. Later 
(A)

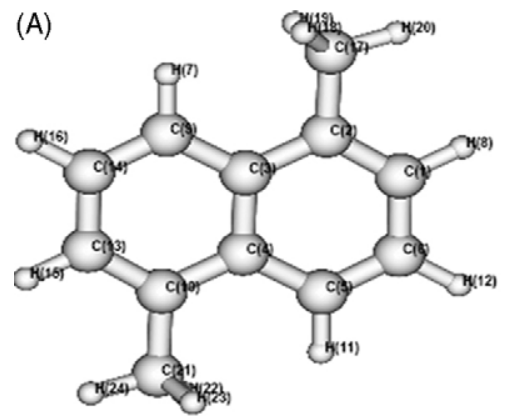

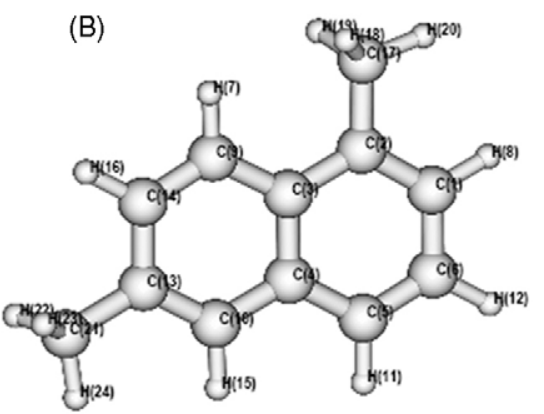

Fig. 1. Optimized structures of 1,5-DMN (A), 1,6-DMN (B) and 2,6-DMN (C) at B3LYP/6-31G* level.

Joblin et al. recorded the IR spectra of neutral PAHs in the gas phase using an oven made from nickel-chromium alloy and fitted with IR transparent diamond windows $[12,13]$. We have used a variable long path-length cell (Model 7.2-V, REFLEX Analytical Corporation) and ultra high pure (UHP) argon as carrier gas for recording the IR spectra of DMNs. The variable path-length cell body is a cylinder of borosilicate glass of length $20 \mathrm{~cm}$, with an inside diameter of $6 \mathrm{~cm}$. It has an optical path adjustable from 1.2 to $7.2 \mathrm{~m}$ in increments of $0.6 \mathrm{~m}$. The cell contains three internal gold-coated mirrors. Of these, two are immovable and one is movable. Two potassium chloride windows of $2.5 \mathrm{~cm}$ diameter are used for the entrance and exit of IR radiation into the cell. In addition to this there are two plane transfer mirrors, which couple the cell with the spectrometer optical path. The cell can be evacuated by a pump and the sample holder is connected through a valve to the cell. The sample DMN vapour mixed with Ar is introduced into the long path-length cell which includes the sample holder is maintained at $90{ }^{\circ} \mathrm{C}$.

The DMNs were subjected to a repeated freeze/pump thaw procedure before use. The total pressure of argon gas containing DMNs in the cell was maintained at $\sim 25$ Torr. Mid-infrared spectra (4000-400 $\left.\mathrm{cm}^{-1}\right)$ were collected using a deuterated triglycine sulphate (DTGS) detector $/ \mathrm{KBr}$ beam splitter combination at $0.5 \mathrm{~cm}^{-1}$ resolution with averaging over 2048 scans.

The quantity of interest in the intensity measurements is the integrated absorption coefficient $A$ (in $\mathrm{cm}^{-2} \mathrm{~atm}^{-1}$ ) which can be determined using the following expression given by Galabov and Dudev [14]:

$A=\frac{2.303 \int \log \left(I_{0} / I\right) \mathrm{d} v}{P l}$

where $P$ is the pressure (in atm) of the compound and $l$ is the optical path length (in $\mathrm{cm}$ ). In our experiments it is not possible to obtain the pressure of the DMNs since they are seeded with a carrier gas and their partial pressures at an elevated temperature in the mixture is unknown. Therefore, we have calculated the integrated band areas $\int \log \left(I_{0} / I\right) \mathrm{d} \nu\left(\right.$ in $\left.^{-1}\right)$ with the help of OMNIC software provided by Thermonicolet under all the experimental bands and obtain pressure of the DMN from Eq. (1) using the calculated absolute intensities. The average pressure obtained from all the bands using the above procedure is used to calculate absolute experimental intensities. In order to get the absolute experimental IR intensities in $\mathrm{km} \mathrm{mol}^{-1}$, the values in $\mathrm{cm}^{-2} \mathrm{~atm}^{-1}$ were multiplied by factor of $82.056(\mathrm{Tl}$ $K)$.

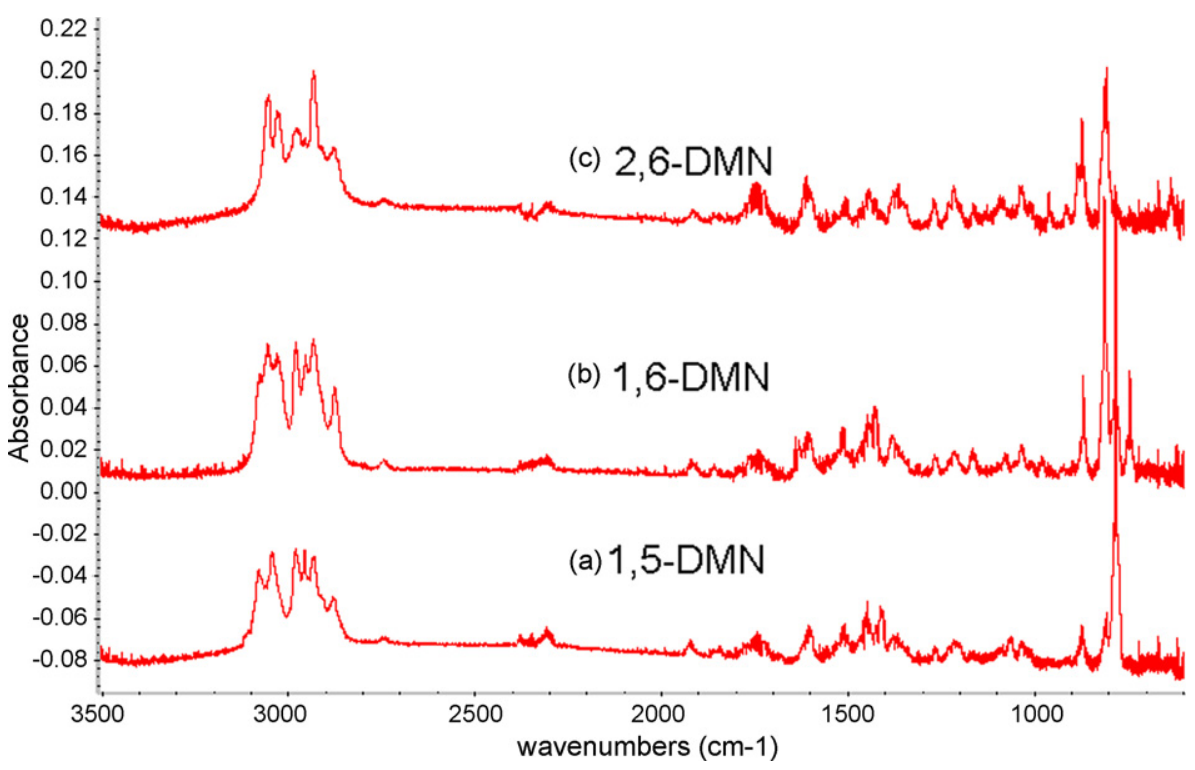

Fig. 2. Enlarged experimental IR absorption spectra for (a) 1,5-DMN, (b) 1,6-DMN, and (c) 2,6-DMN at $0.5 \mathrm{~cm}^{-1}$ resolution. 
Theoretical calculations were carried out using density functional theory (DFT) to determine the harmonic frequencies and intensities of 1,6-DMN, 1,5-DMN and 2,6-DMN. We have followed the approach that has been used by Bauschlicher et al. $[8,9]$, where they performed DFT calculations with the B3LYP/ 6-31G* and B3LYP/4-31G level using the Gaussian 94 codes. In our case, geometry optimization was carried out with standard polarization basis sets $6-31 G^{*}$ at the B3LYP (Becke 1993; Lee, Yang, and Parr 1988) level of theory using Gaussian 98 [15] codes in an IBM RS/6000 computers.

The frequencies and intensities were determined at the fully optimized $C_{1}$ geometry (Fig. 1). From the Gaussian output results we could predict the spectra for these compounds. For each spectral line full-width at half-maximum (FWHM) was assumed to be $15 \mathrm{~cm}^{-1}$ and the calculated frequencies were scaled by a factor of 0.9588 for $\mathrm{C}-\mathrm{H}$ stretch and 0.9733 for other modes to compare with experiment [16]. It was reported by Langhoff et al. [17] that many of the methyl PAHs are found to be of lower symmetry $\left(C_{1}\right)$ due to slight rotation of the methyl group, although the true point group of the molecule is $C_{\mathrm{s}}$. In our B3LYP calculations on DMNs, we found at least one imaginary frequency at the $C_{\mathrm{s}}$ optimized structure and thus $C_{1}$ symmetry was chosen for calculation.

\section{Results and discussion}

The gas phase IR spectra of the three DMNs investigated in this report are shown in Fig. 2 and the experimental and theoretical infrared frequencies and intensities are listed in Tables 1-3. Absolute intensities are listed first while relative intensities are shown in parentheses. The relative intensities are given with respect to the strongest band seen in the range 780 $815 \mathrm{~cm}^{-1}$. The bands observed at 783.3, 812.8 and $808.5 \mathrm{~cm}^{-1}$ in 1,5-, 1,6-, and 2,6-DMN, respectively, correlate with the calculated fundamentals at 786.5,812.4, and $811.6 \mathrm{~cm}^{-1}$ which is assigned to a $\operatorname{Ar}(\mathrm{C}-\mathrm{H})$ out-of-plane bending mode. To

Table 1

Calculated and experimental infrared frequencies $\left(\mathrm{cm}^{-1}\right)$ and intensities in $\mathrm{km} \mathrm{mol}^{-1}$ for $1,5-\mathrm{DMN}$

\begin{tabular}{|c|c|c|c|c|c|c|}
\hline \multirow[t]{2}{*}{ No. } & \multicolumn{2}{|l|}{ Theoretical } & \multicolumn{2}{|c|}{ Experimental } & \multirow{2}{*}{$\begin{array}{l}\text { NIST data }^{\mathrm{a}} \\
\text { Frequency } \\
\text { (relative intensity) }^{\mathrm{c}}\end{array}$} & \multirow[t]{2}{*}{ Mode of vibrations } \\
\hline & Frequency $^{\mathrm{b}}$ & $\begin{array}{l}\text { Intensity } \\
\text { (relative intensity) }\end{array}$ & Frequency & $\begin{array}{l}\text { Intensity } \\
\text { (relative intensity) }\end{array}$ & & \\
\hline 1 & 93.89 & $0.34(0.005)$ & & & & \\
\hline 2 & 162.66 & $0.00(0.000)$ & & & & \\
\hline 3 & 182.99 & $0.00(0.000)$ & & & & \\
\hline 4 & 190.28 & $2.27(0.034)$ & & & & \\
\hline 5 & 202.17 & $0.00(0.000)$ & & & & \\
\hline 6 & 253.25 & $0.44(0.006)$ & & & & \\
\hline 7 & 263.59 & $3.76(0.056)$ & & & & \\
\hline 8 & 323.01 & $0.00(0.000)$ & & & & \\
\hline 9 & 453.84 & $0.00(0.000)$ & & & & \\
\hline 10 & 455.30 & $0.00(0.000)$ & & & & \\
\hline 11 & 469.75 & $0.00(0.000)$ & & & & \\
\hline 12 & 479.88 & $1.23(0.018)$ & & & & \\
\hline 13 & 504.95 & $0.00(0.000)$ & & & & \\
\hline 14 & 521.55 & $5.30(0.079)$ & & & & \\
\hline 15 & 567.17 & $0.89(0.013)$ & & & & \\
\hline 16 & 626.00 & $0.00(0.000)$ & & & & \\
\hline 17 & 628.42 & $0.00(0.000)$ & & & & \\
\hline 18 & 730.99 & $0.00(0.000)$ & & & & \\
\hline 19 & 777.75 & $2.48(0.037)$ & & & & \\
\hline 20 & 786.50 & $66.58(1.000)$ & 783.3 & $61.42(1.000)$ & $781.9(1.000)$ & $\alpha_{\operatorname{Ar}(\mathrm{C}-\mathrm{H})}$ \\
\hline 21 & 802.06 & $0.00(0.000)$ & & & & \\
\hline 22 & 842.89 & $0.00(0.000)$ & & & & \\
\hline 23 & 869.65 & $1.39(0.020)$ & 807.5 & $5.89(0.096)$ & & $\alpha_{\mathrm{Ar}(\mathrm{C}-\mathrm{H})}$ \\
\hline 24 & 877.85 & $0.36(0.005)$ & & & & \\
\hline 25 & 889.48 & $0.00(0.000)$ & & & & \\
\hline 26 & 945.88 & $1.11(0.016)$ & 872.2 & $6.24(0.101)$ & $873.9(0.042)$ & $\alpha_{\operatorname{Ar}(\mathrm{C}-\mathrm{H})}$ \\
\hline 27 & 947.32 & $0.00(0.000)$ & & & & \\
\hline 28 & 985.96 & $0.00(0.000)$ & & & & \\
\hline 29 & 1015.13 & $2.20(0.033)$ & & & & \\
\hline 30 & 1047.22 & $0.00(0.000)$ & & & & \\
\hline 31 & 1048.50 & $4.12(0.061)$ & 1034.7 & $2.42(0.039)$ & & $\beta_{\mathrm{Me}(\mathrm{C}-\mathrm{H})}$ \\
\hline 32 & 1069.71 & $6.49(0.097)$ & 1062.6 & $4.16(0.067)$ & $1064.1(0.075)$ & $\gamma_{\text {Ar-ring }}$ \\
\hline 33 & 1100.39 & $0.00(0.000)$ & & & & \\
\hline 34 & 1135.14 & $0.00(0.000)$ & & & & \\
\hline 35 & 1174.00 & $1.19(0.017)$ & & & & \\
\hline 36 & 1174.16 & $0.00(0.000)$ & & & & \\
\hline 37 & 1218.38 & $2.76(0.041)$ & & & & \\
\hline 38 & 1233.35 & $0.00(0.000)$ & & & & \\
\hline
\end{tabular}


Table 1 (Continued)

\begin{tabular}{|c|c|c|c|c|c|c|}
\hline \multirow[t]{2}{*}{ No. } & \multicolumn{2}{|l|}{ Theoretical } & \multicolumn{2}{|c|}{ Experimental } & \multirow{2}{*}{$\begin{array}{l}\text { NIST data }^{\mathrm{a}} \\
\text { Frequency } \\
\text { (relative intensity) }^{\mathrm{c}}\end{array}$} & \multirow[t]{2}{*}{ Mode of vibrations } \\
\hline & Frequency $^{\mathrm{b}}$ & $\begin{array}{l}\text { Intensity } \\
\text { (relative intensity) }\end{array}$ & Frequency & $\begin{array}{l}\text { Intensity } \\
\text { (relative intensity) }\end{array}$ & & \\
\hline 39 & 1258.63 & $2.43(0.036)$ & $\begin{array}{l}1217.3 \\
1264.0\end{array}$ & $\begin{array}{l}2.08(0.033) \\
1.73(0.028)\end{array}$ & & $\delta_{\mathrm{Ar}(\mathrm{C}-\mathrm{H})}+\gamma_{\text {Ar-ring }}$ \\
\hline $\begin{array}{l}40 \\
41 \\
42 \\
43\end{array}$ & $\begin{array}{l}1348.38 \\
1362.95 \\
1403.11 \\
1404.75\end{array}$ & $\begin{array}{l}0.92(0.013) \\
0.00(0.000) \\
0.00(0.000) \\
0.48(0.007)\end{array}$ & & & & \\
\hline $\begin{array}{l}44 \\
45 \\
46\end{array}$ & $\begin{array}{l}1416.24 \\
1416.69 \\
1472.51\end{array}$ & $\begin{array}{r}17.25(0.259) \\
0.00(0.000) \\
0.00(0.000)\end{array}$ & 1413.9 & $6.24(0.101)$ & $1410.2(0.173)$ & $\delta_{\mathrm{Ar}(\mathrm{C}-\mathrm{H})}$ \\
\hline $\begin{array}{l}47 \\
48 \\
49 \\
50\end{array}$ & $\begin{array}{l}1474.06 \\
1474.19 \\
1486.78 \\
1494.33\end{array}$ & $\begin{array}{r}6.13(0.092) \\
6.60(0.099) \\
18.53(0.278) \\
0.00(0.000)\end{array}$ & 1448.9 & $5.55(0.090)$ & $1451.0(0.068)$ & $\tau_{\mathrm{Me}(\mathrm{C}-\mathrm{H})}$ \\
\hline $\begin{array}{l}51 \\
52\end{array}$ & $\begin{array}{l}1524.04 \\
1598.43\end{array}$ & $\begin{array}{l}8.57(0.128) \\
0.00(0.000)\end{array}$ & 1510.9 & $3.12(0.050)$ & 1510.7 (0.116) & $v_{\mathrm{Ar}(\mathrm{C}-\mathrm{C})}$ \\
\hline 53 & 1615.03 & $12.97(0.194)$ & 1606.1 & $12.83(0.209)$ & $1600.5(0.146)$ & $v_{\mathrm{Ar}(\mathrm{C}-\mathrm{C})}$ \\
\hline 54 & 1629.94 & $0.00(0.000)$ & $\begin{array}{l}1745.3 \\
1848.6 \\
1922.2 \\
2744.6\end{array}$ & $\begin{array}{l}6.59(0.107) \\
2.42(0.039) \\
2.77(0.045) \\
1.38(0.022)\end{array}$ & $\begin{array}{l}1681.8(0.040) \\
1774.1(0.034) \\
1848.8(0.044) \\
1920.0(0.608)\end{array}$ & \\
\hline 55 & 2913.59 & $58.53(0.879)$ & & & & \\
\hline $\begin{array}{l}56 \\
57\end{array}$ & $\begin{array}{l}2913.92 \\
2961.12\end{array}$ & $\begin{array}{r}1.66(0.024) \\
12.25(0.183)\end{array}$ & 2882.4 & $9.02(0.146)$ & $2882.6(0.043)$ & $\chi_{\mathrm{Me}(\mathrm{C}-\mathrm{H}) \mathrm{Sym}}$ \\
\hline $\begin{array}{l}58 \\
59\end{array}$ & $\begin{array}{l}2961.25 \\
2995.98\end{array}$ & $\begin{array}{l}29.00(0.435) \\
18.16(0.272)\end{array}$ & 2931.8 & $6.59(0.107)$ & $2938.6(0.055)$ & $\chi_{\mathrm{Me}(\mathrm{C}-\mathrm{H}) \text { Asym }}$ \\
\hline $\begin{array}{l}60 \\
61\end{array}$ & $\begin{array}{l}2997.18 \\
3045.84\end{array}$ & $\begin{array}{l}19.24(0.288) \\
25.83(0.387)\end{array}$ & 2956.9 & $3.12(0.050)$ & $2957.9(0.012)$ & $\chi_{\mathrm{Me}(\mathrm{C}-\mathrm{H}) \text { Asym }}$ \\
\hline $\begin{array}{l}62 \\
63\end{array}$ & $\begin{array}{l}3046.36 \\
3062.31\end{array}$ & $\begin{array}{l}0.00(0.000) \\
0.16(0.002)\end{array}$ & 2979.4 & $11.10(0.180)$ & $2977.2(0.085)$ & $\Pi_{\mathrm{Ar}(\mathrm{C}-\mathrm{H})}$ \\
\hline $\begin{array}{l}64 \\
65\end{array}$ & $\begin{array}{l}3062.45 \\
3083.33\end{array}$ & $\begin{array}{l}49.13(0.737) \\
41.41(0.621)\end{array}$ & 3043.2 & $15.26(0.248)$ & $3041.3(0.175)$ & $\Pi_{\mathrm{Ar}(\mathrm{C}-\mathrm{H})}$ \\
\hline 66 & 3083.79 & $0.03(0.000)$ & 3081.0 & $10.06(0.163)$ & $3079.9(0.252)$ & $\Pi_{\mathrm{Ar}(\mathrm{C}-\mathrm{H})}$ \\
\hline
\end{tabular}

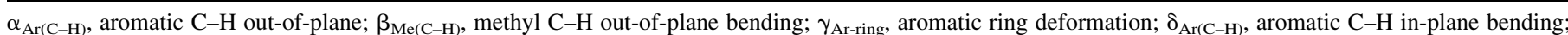

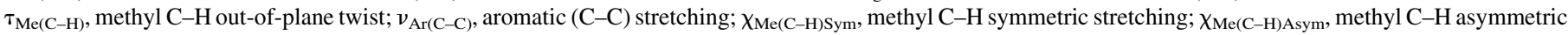
stretching; $\Pi_{\mathrm{Ar}(\mathrm{C}-\mathrm{H})}$, aromatic $\mathrm{C}-\mathrm{H}$ stretching.

a Adapted from Ref. [10].

b The B3LYP/6-31G* frequencies are scaled by 0.9588 for $\mathrm{C}-\mathrm{H}$ stretching and 0.9733 for other modes [16].

${ }^{c}$ In case of NIST reported spectra, only relative intensities are given in parentheses.

Table 2

Calculated and experimental infrared frequencies $\left(\mathrm{cm}^{-1}\right)$ and intensities in $\mathrm{km} \mathrm{mol}^{-1}$ for 1,6-DMN

\begin{tabular}{|c|c|c|c|c|c|}
\hline \multirow[t]{2}{*}{ No. } & \multicolumn{2}{|l|}{ Theoretical } & \multicolumn{2}{|c|}{ Experimental } & \multirow[t]{2}{*}{ Mode of vibrations } \\
\hline & Frequency $^{\mathrm{a}}$ & Intensity (relative intensity) & Frequency & Intensity (relative intensity) & \\
\hline 1 & 88.72 & $0.51(0.016)$ & & & \\
\hline 2 & 107.00 & $0.47(0.014)$ & & & \\
\hline 3 & 148.54 & $0.11(0.003)$ & & & \\
\hline 4 & 189.46 & $0.69(0.022)$ & & & \\
\hline 5 & 233.38 & $0.61(0.019)$ & & & \\
\hline 6 & 247.55 & $1.71(0.054)$ & & & \\
\hline 7 & 285.04 & $0.22(0.007)$ & & & \\
\hline 8 & 328.34 & $0.05(0.001)$ & & & \\
\hline 9 & 426.34 & $3.04(0.096)$ & & & \\
\hline 10 & 452.48 & $0.13(0.004)$ & & & \\
\hline 11 & 463.72 & $0.89(0.028)$ & & & \\
\hline 12 & 494.88 & $2.61(0.083)$ & & & \\
\hline 13 & 509.77 & $2.15(0.068)$ & & & \\
\hline
\end{tabular}


Table 2 (Continued)

\begin{tabular}{|c|c|c|c|c|c|}
\hline \multirow[t]{2}{*}{ No. } & \multicolumn{2}{|l|}{ Theoretical } & \multicolumn{2}{|l|}{ Experimental } & \multirow[t]{2}{*}{ Mode of vibrations } \\
\hline & Frequency $^{\mathrm{a}}$ & Intensity (relative intensity) & Frequency & Intensity (relative intensity) & \\
\hline 14 & 561.05 & $0.39(0.012)$ & & & \\
\hline 15 & 584.63 & $1.03(0.032)$ & & & \\
\hline 16 & 642.55 & $0.66(0.021)$ & & & \\
\hline 17 & 703.38 & $1.03(0.033)$ & & & \\
\hline 18 & 710.37 & $0.64(0.020)$ & & & \\
\hline 19 & 747.33 & $5.93(0.188)$ & 746.2 & $10.02(0.352)$ & $\alpha_{\mathrm{Ar}(\mathrm{C}-\mathrm{H})}$ \\
\hline 20 & 784.54 & $14.14(0.449)$ & 784.1 & $13.49(0.474)$ & $\alpha_{\operatorname{Ar}(\mathrm{C}-\mathrm{H})}$ \\
\hline 21 & 812.42 & $31.45(1.000)$ & 812.8 & $28.42(1.000)$ & $\alpha_{\mathrm{Ar}(\mathrm{C}-\mathrm{H})}$ \\
\hline 22 & 823.60 & $0.68(0.021)$ & & & \\
\hline 23 & 867.99 & $6.73(0.214)$ & & & \\
\hline 24 & 893.11 & $2.80(0.089)$ & 870.2 & $8.99(0.316)$ & $\alpha_{\mathrm{Ar}(\mathrm{C}-\mathrm{H})}$ \\
\hline 25 & 921.59 & $0.41(0.013)$ & & & \\
\hline 26 & 939.58 & $0.17(0.005)$ & & & \\
\hline 27 & 946.68 & $0.91(0.028)$ & & & \\
\hline 28 & 976.12 & $2.71(0.086)$ & & & \\
\hline 29 & 1009.17 & $5.66(0.179)$ & 981.9 & $1.84(0.064)$ & $\sigma_{\mathrm{Me}(\mathrm{C}-\mathrm{H})}$ \\
\hline 30 & 1048.29 & $5.39(0.171)$ & & & \\
\hline 31 & 1048.44 & $1.09(0.034)$ & 1038.1 & $2.65(0.093)$ & $\beta_{\mathrm{Me}(\mathrm{C}-\mathrm{H})}$ \\
\hline 32 & 1051.11 & $0.85(0.027)$ & & & \\
\hline 33 & 1080.50 & $2.64(0.084)$ & 1076.0 & $2.45(0.086)$ & $\delta_{\mathrm{Ar}(\mathrm{C}-\mathrm{H})}$ \\
\hline 34 & 1162.61 & $0.56(0.018)$ & & & \\
\hline 35 & 1167.46 & $1.00(0.032)$ & & & \\
\hline 36 & 1176.66 & $2.59(0.082)$ & 1167.3 & $2.65(0.093)$ & $\delta_{\mathrm{Ar}(\mathrm{C}-\mathrm{H})}+\gamma_{\mathrm{Ar}-\text { ring }}$ \\
\hline 37 & 1220.44 & $1.71(0.054)$ & 1217.5 & $2.86(0.101)$ & $\delta_{\mathrm{Ar}(\mathrm{C}-\mathrm{H})}+\gamma_{\mathrm{Ar}-\mathrm{ring}}$ \\
\hline 38 & 1248.59 & $0.30(0.009)$ & & & \\
\hline 39 & 1268.85 & $3.18(0.101)$ & 1269.7 & $2.86(0.101)$ & $\delta_{\mathrm{Ar}(\mathrm{C}-\mathrm{H})}+\gamma_{\mathrm{Ar}-\mathrm{ring}}$ \\
\hline 40 & 1357.95 & $1.45(0.046)$ & & & \\
\hline 41 & 1373.18 & $2.03(0.064)$ & & & \\
\hline 42 & 1378.15 & $2.14(0.068)$ & & & \\
\hline 43 & 1404.22 & $0.77(0.024)$ & 1380.4 & $10.63(0.374)$ & $\sigma_{\mathrm{Me}(\mathrm{C}-\mathrm{H})}$ \\
\hline 44 & 1405.16 & $0.47(0.015)$ & & & \\
\hline 45 & 1437.97 & $9.57(0.304)$ & 1426.5 & $5.93(0.208)$ & $\delta_{\mathrm{Ar}(\mathrm{C}-\mathrm{H})}$ \\
\hline 46 & 1467.29 & $2.90(0.092)$ & & & \\
\hline 47 & 1473.55 & $4.16(0.132)$ & & & \\
\hline 48 & 1474.13 & $7.79(0.247)$ & 1450.0 & $3.68(0.129)$ & $\tau_{\mathrm{Me}(\mathrm{C}-\mathrm{H})}$ \\
\hline 49 & 1485.63 & $4.28(0.136)$ & & & \\
\hline 50 & 1494.07 & $4.32(0.137)$ & & & \\
\hline 51 & 1520.97 & $9.85(0.313)$ & 1513.6 & $7.56(0.266)$ & $v_{\mathrm{Ar}(\mathrm{C}-\mathrm{C})}$ \\
\hline 52 & 1590.91 & $1.35(0.043)$ & & & \\
\hline 53 & 1618.56 & $11.07(0.352)$ & 1605.8 & $5.72(0.201)$ & $v_{\mathrm{Ar}(\mathrm{C}-\mathrm{C})}$ \\
\hline 54 & 1642.67 & $8.73(0.277)$ & 1638.6 & $4.08(0.143)$ & $v_{\mathrm{Ar}(\mathrm{C}-\mathrm{C})}$ \\
\hline & & 1741.0 & $9.2(0.323)$ & & \\
\hline & & 1858.9 & $1.63(0.057)$ & & \\
\hline & & 1922.4 & $3.88(0.136)$ & & \\
\hline & & 2746.6 & $2.04(0.072)$ & & \\
\hline 55 & 2913.63 & $35.41(1.126)$ & & & \\
\hline 56 & 2914.28 & $35.11(1.116)$ & 2874.8 & $11.24(0.395)$ & $\chi_{\mathrm{Me}(\mathrm{C}-\mathrm{H}) \mathrm{Sym}}$ \\
\hline 57 & 2960.69 & $9.81(0.312)$ & & & \\
\hline 58 & 2961.83 & $23.10(0.734)$ & 2933.3 & $11.24(0.395)$ & $\chi_{\mathrm{Me}(\mathrm{C}-\mathrm{H}) \mathrm{Asym}}$ \\
\hline 59 & 2997.16 & $16.61(0.528)$ & & & \\
\hline 60 & 2997.86 & $18.91(0.601)$ & 2953.8 & $2.24(0.079)$ & $\chi_{\mathrm{Me}(\mathrm{C}-\mathrm{H}) \text { Asym }}$ \\
\hline 61 & 3040.66 & $16.86(0.536)$ & 2980.5 & $11.45(0.402)$ & $\Pi_{\mathrm{Ar}(\mathrm{C}-\mathrm{H})}$ \\
\hline 62 & 3044.43 & $14.64(0.465)$ & & & \\
\hline 63 & 3045.16 & $0.76(0.024)$ & 3031.8 & $7.77(0.273)$ & $\Pi_{\mathrm{Ar}(\mathrm{C}-\mathrm{H})}$ \\
\hline 64 & 3052.60 & $30.16(0.958)$ & & & \\
\hline 65 & 3070.49 & 37.47 (1.191) & 3056.8 & $4.49(0.158)$ & $\Pi_{\mathrm{Ar}(\mathrm{C}-\mathrm{H})}$ \\
\hline 66 & 3077.56 & $15.71(0.499)$ & 3078.0 & $3.68(0.129)$ & $\Pi_{\mathrm{Ar}(\mathrm{C}-\mathrm{H})}$ \\
\hline
\end{tabular}

$\alpha_{\mathrm{Ar}(\mathrm{C}-\mathrm{H})}$, aromatic $\mathrm{C}-\mathrm{H}$ out-of-plane bending; $\sigma_{\mathrm{Me}(\mathrm{C}-\mathrm{H})}$, methyl $\mathrm{C}-\mathrm{H}$ in-plane bending; $\beta_{\mathrm{Me}(\mathrm{C}-\mathrm{H})}$, methyl C-H out-of-plane bending; $\gamma_{\mathrm{Ar}-\mathrm{ring}}$, aromatic ring deformation, $\delta_{\mathrm{Ar}(\mathrm{C}-\mathrm{H})}$, aromatic $\mathrm{C}-\mathrm{H}$ in-plane bending; $\tau_{\mathrm{Me}(\mathrm{C}-\mathrm{H})}$, methyl $\mathrm{C}-\mathrm{H}$ out-of-plane twist; $\nu_{\mathrm{Ar}(\mathrm{C}-\mathrm{C})}$, aromatic $(\mathrm{C}-\mathrm{C})$ stretching; $\chi_{\mathrm{Me}(\mathrm{C}-\mathrm{H}) \mathrm{Sym}}, \mathrm{methyl} \mathrm{C-H}$ symmetric stretching; $\chi_{\mathrm{Me}(\mathrm{C}-\mathrm{H}) \mathrm{Asym}}$, methyl $\mathrm{C}-\mathrm{H}$ asymmetric stretching; $\Pi_{\mathrm{Ar}(\mathrm{C}-\mathrm{H})}$, aromatic $\mathrm{C}-\mathrm{H}$ stretching.

${ }^{a}$ The B3LYP/6-31G* frequencies are scaled by 0.9588 for $\mathrm{C}-\mathrm{H}$ stretching and 0.9733 for other modes [16]. 
Table 3

Calculated and experimental infrared frequencies $\left(\mathrm{cm}^{-1}\right)$ and intensities in $\mathrm{km} \mathrm{mol}^{-1}$ for 2,6-DMN

\begin{tabular}{|c|c|c|c|c|c|c|}
\hline \multirow[t]{2}{*}{ No. } & \multicolumn{2}{|l|}{ Theoretical } & \multicolumn{2}{|c|}{ Experimental } & \multirow{2}{*}{$\begin{array}{l}\text { NIST Data }^{\mathrm{a}} \\
\text { Frequency } \\
\text { (relative intensity) }^{\mathrm{c}}\end{array}$} & \multirow[t]{2}{*}{ Mode of Vibrations } \\
\hline & Frequency $^{b}$ & $\begin{array}{l}\text { Intensity } \\
\text { (relative intenstity) }\end{array}$ & Frequency & $\begin{array}{l}\text { Intensity } \\
\text { (relative intenstiy) }\end{array}$ & & \\
\hline 1 & 76.86 & $1.16(0.037)$ & & & & \\
\hline 2 & 87.45 & $0.00(0.000)$ & & & & \\
\hline 3 & 95.69 & $0.04(0.001)$ & & & & \\
\hline 4 & 180.07 & $0.39(0.012)$ & & & & \\
\hline 5 & 201.25 & $0.00(0.000)$ & & & & \\
\hline 6 & 215.90 & $0.88(0.028)$ & & & & \\
\hline 7 & 313.50 & $0.00(0.000)$ & & & & \\
\hline 8 & 333.87 & $2.91(0.093)$ & & & & \\
\hline 9 & 395.74 & $0.00(0.000)$ & & & & \\
\hline 10 & 402.16 & $0.00(0.000)$ & & & & \\
\hline 11 & 429.88 & $2.31(0.074)$ & & & & \\
\hline 12 & 482.68 & $5.94(0.189)$ & & & & \\
\hline 13 & 521.53 & $0.00(0.000)$ & & & & \\
\hline 14 & 525.00 & $0.00(0.000)$ & & & & \\
\hline 15 & 574.59 & $1.21(0.038)$ & & & & \\
\hline 16 & 632.80 & $3.40(0.108)$ & & & & \\
\hline 17 & 668.29 & $1.35(0.043)$ & & & & \\
\hline 18 & 747.20 & $0.00(0.000)$ & & & & \\
\hline 19 & 757.53 & $0.00(0.000)$ & & & & \\
\hline 20 & 789.89 & $0.00(0.000)$ & & & & \\
\hline 21 & 811.61 & $31.33(1.000)$ & 808.5 & $33.33(1.000)$ & $807.3(1.00)$ & $\alpha_{\mathrm{Ar}(\mathrm{C}-\mathrm{H})}$ \\
\hline 22 & 820.21 & $0.00(0.000)$ & & & & \\
\hline 23 & 874.72 & $22.41(0.715)$ & 873.6 & $22.91(0.687)$ & $870.7(0.61)$ & $\alpha_{\mathrm{Ar}(\mathrm{C}-\mathrm{H})}$ \\
\hline 24 & 884.62 & $0.00(0.000)$ & & & & \\
\hline 25 & 911.05 & $0.98(0.031)$ & & & & \\
\hline 26 & 940.49 & $0.94(0.030)$ & 914.2 & $2.50(0.075)$ & & $\alpha_{\operatorname{Ar}(\mathrm{C}-\mathrm{H})}$ \\
\hline 27 & 941.29 & $0.00(0.000)$ & & & & \\
\hline 28 & 957.44 & $0.00(0.000)$ & & & & \\
\hline 29 & 1004.40 & $0.00(0.000)$ & & & & \\
\hline 30 & 1012.35 & $13.82(0.441)$ & 961.4 & $1.66(0.049)$ & & $\sigma_{\mathrm{Me}(\mathrm{C}-\mathrm{H})}$ \\
\hline 31 & 1047.62 & $8.56(0.273)$ & 1037.9 & $8.33(0.249)$ & & $\beta_{\mathrm{Me}(\mathrm{C}-\mathrm{H})}$ \\
\hline 32 & 1048.39 & $0.01(0.000)$ & & & & \\
\hline 33 & 1132.27 & $1.83(0.058)$ & 1093.1 & $4.16(0.124)$ & & $\delta_{\mathrm{Ar}(\mathrm{C}-\mathrm{H})}$ \\
\hline 34 & 1151.69 & $0.00(0.000)$ & & & & \\
\hline 35 & 1170.53 & $3.95(0.126)$ & & & & \\
\hline 36 & 1179.34 & $0.00(0.000)$ & & & & \\
\hline 37 & 1220.40 & $1.02(0.032)$ & & & & \\
\hline 38 & 1258.61 & $0.00(0.000)$ & & & & \\
\hline \multirow[t]{2}{*}{39} & 1267.74 & $5.22(0.166)$ & 1216.4 & $13.33(0.399)$ & & $\delta_{\mathrm{Ar}(\mathrm{C}-\mathrm{H})}+\gamma_{\text {Ar-ring }}$ \\
\hline & & & 1273.0 & $5.00(0.150)$ & & \\
\hline 40 & 1348.64 & $1.69(0.054)$ & & & & \\
\hline 41 & 1383.14 & $0.00(0.000)$ & & & & \\
\hline 42 & 1386.19 & $5.58(0.178)$ & 1365.0 & $15.00(0.450)$ & & $\delta_{\mathrm{Ar}(\mathrm{C}-\mathrm{H})}$ \\
\hline 43 & 1405.15 & $1.38(0.044)$ & & & & \\
\hline 44 & 1405.60 & $0.01(0.000)$ & & & & \\
\hline 45 & 1412.03 & $0.00(0.000)$ & & & & \\
\hline 46 & 1472.61 & $7.38(0.235)$ & & & & \\
\hline 47 & 1472.76 & $3.47(0.110)$ & 1450.1 & $7.50(0.225)$ & $1449.9(0.113)$ & $\tau_{\mathrm{Me}(\mathrm{C}-\mathrm{H})}$ \\
\hline 48 & 1476.34 & $11.15(0.356)$ & & & & \\
\hline 49 & 1485.87 & $0.00(0.000)$ & & & & \\
\hline 50 & 1495.08 & $0.00(0.000)$ & & & & \\
\hline 51 & 1518.62 & $11.66(0.372)$ & 1510.9 & $5.83(0.175)$ & $1504.7(0.194)$ & $v_{\mathrm{Ar}(\mathrm{C}-\mathrm{C})}$ \\
\hline 52 & 1582.20 & $0.00(0.000)$ & & & & \\
\hline 53 & 1627.21 & $25.93(0.827)$ & 1612.9 & $20.00(0.600)$ & $1611.3(0.714)$ & $v_{\mathrm{Ar}(\mathrm{C}-\mathrm{C})}$ \\
\hline \multirow[t]{4}{*}{54} & 1648.64 & $0.00(0.000)$ & & & & \\
\hline & & & 1741.8 & $21.25(0.637)$ & $1745.3(0.125)$ & \\
\hline & & & 1908.1 & $1.66(0.049)$ & $1906.0(0.109)$ & \\
\hline & & & 2744.5 & $2.08(0.062)$ & & \\
\hline
\end{tabular}


Table 3 (Continued)

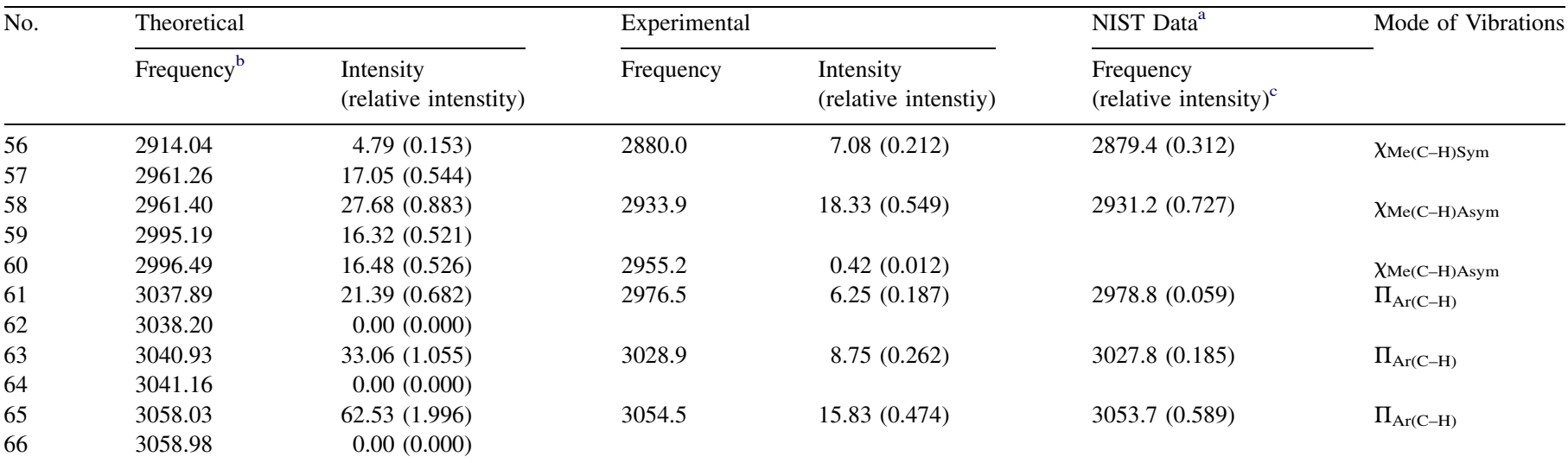

$\alpha_{\mathrm{Ar}(\mathrm{C}-\mathrm{H})}$, aromatic $\mathrm{C}-\mathrm{H}$ out-of-plan bending; $\sigma_{\mathrm{Me}(\mathrm{C}-\mathrm{H})}$, methyl $\mathrm{C}-\mathrm{H}$ in-plane bending; $\beta_{\mathrm{Me}(\mathrm{C}-\mathrm{H})}$, methyl C-H out-of-plane bending; $\gamma_{\mathrm{Ar}-\mathrm{ring}}$, aromatic ring deformation; $\delta_{\mathrm{Ar}(\mathrm{C}-\mathrm{H})}$, aromatic $\mathrm{C}-\mathrm{H}$ in-plane bending; $\tau_{\mathrm{Me}(\mathrm{C}-\mathrm{H})}$, methyl $\mathrm{C}-\mathrm{H}$ out-of-plane twist, $\nu_{\mathrm{Ar}(\mathrm{C}-\mathrm{C})}$, aromatic $(\mathrm{C}-\mathrm{C})$ stretching; $\chi_{\mathrm{Me}(\mathrm{C}-\mathrm{H}) \mathrm{Sym}}, \mathrm{methyl} \mathrm{C}-\mathrm{H}$ symmetric stretching; $\chi_{\mathrm{Me}(\mathrm{C}-\mathrm{H}) \text { Asym }}$, methyl $\mathrm{C}-\mathrm{H}$ asymmetric stretching; $\Pi_{\mathrm{Ar}(\mathrm{C}-\mathrm{H})}$, aromatic $\mathrm{C}-\mathrm{H}$ stretching.

${ }^{\text {a }}$ Adapted from Ref. [10].

b The B3LYP/6-31G* frequencies are scaled by 0.9588 for $\mathrm{C}-\mathrm{H}$ stretching and 0.9733 for other modes[16].

${ }^{\mathrm{c}}$ In case of NIST reported spectrum, only relative intensities are given in parentheses.

compare the experimental, calculated and reported [10] spectra, we have displayed all DMN spectra separately in Figs. 3-5. No IR spectrum for 1,6-DMN has been reported and, therefore, literature spectrum of 1-MN has been displayed in Fig. 4 instead. Apart from the intense $\operatorname{Ar}(\mathrm{C}-\mathrm{H})$ out-of-plane bending mode mentioned above, many other low frequency $\operatorname{Ar}(\mathrm{C}-\mathrm{H})$

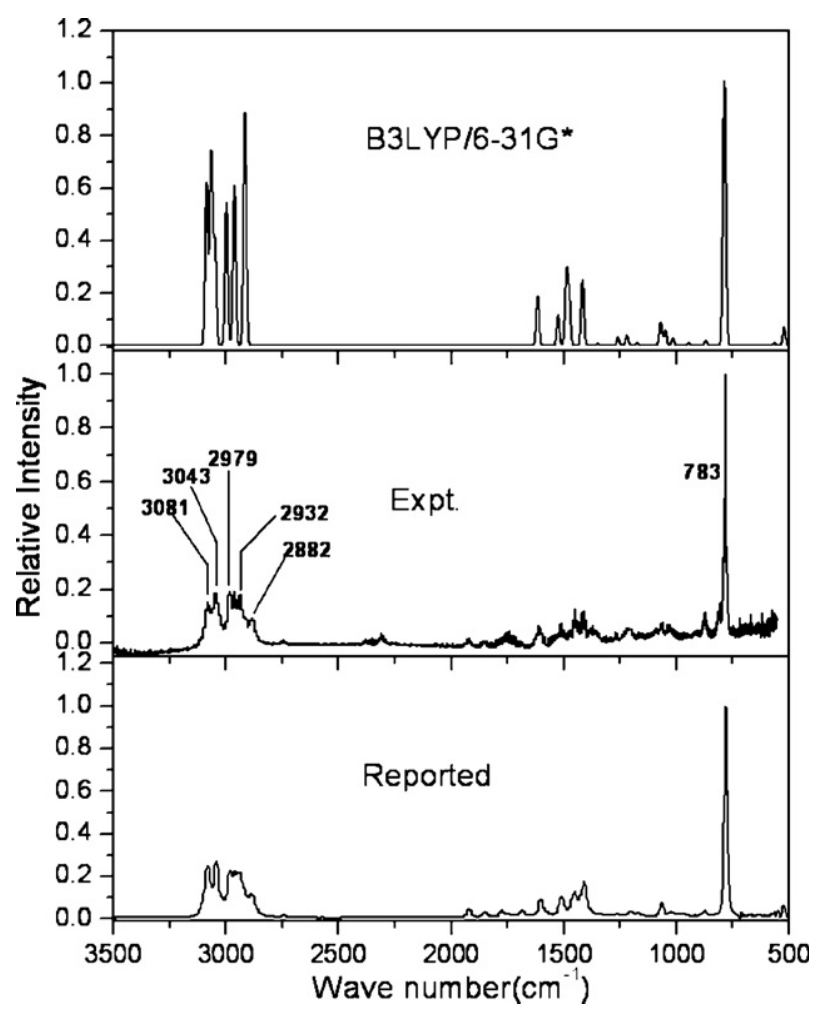

Fig. 3. Comparison of NIST reported data from [10], experimental and theoretical IR absorption spectra of 1,5-DMN. In the calculated spectrum, the FWHM is assumed to be $15 \mathrm{~cm}^{-1}$. Prominent peaks are labeled in the observed spectrum. Very weak bands such as $\mathrm{Me}(\mathrm{C}-\mathrm{H})_{\text {asym stret }}$ at $2956.9 \mathrm{~cm}^{-1}$, etc. are not labeled in the observed spectrum. out-of-plane bending modes with lower intensities have been recorded and assigned in the spectra. Bands observed at 807.5 and $872.2 \mathrm{~cm}^{-1}$ in 1,5-DMN, 746.2, 784.1, and $870.2 \mathrm{~cm}^{-1}$ in 1,6-DMN and 873.6 and $914.2 \mathrm{~cm}^{-1}$ in 2,6-DMN are assigned to $\operatorname{Ar}(\mathrm{C}-\mathrm{H})$ out-of-plane bending vibrations by comparing with the respective calculated bands at 869.6 and $945.8 \mathrm{~cm}^{-1}$ in $1,5-$

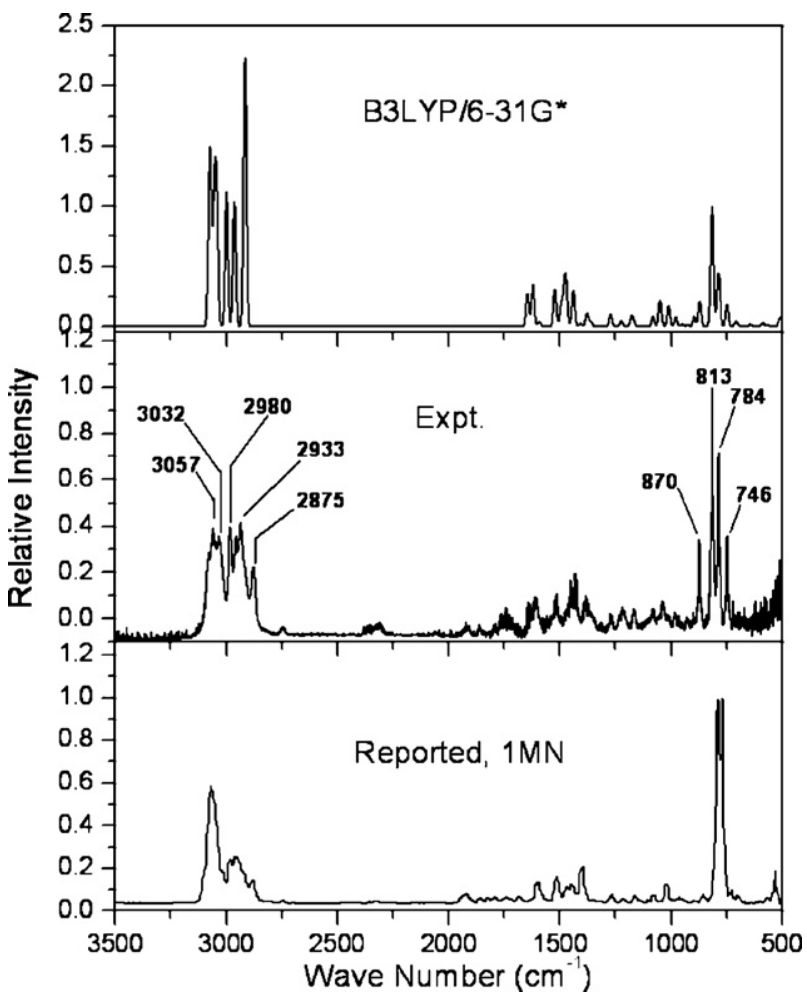

Fig. 4. Comparison of NIST reported spectrum (1-MN) from [10], experimental and calculated IR absorption spectra of 1,6-DMN. In the calculated spectrum the FWHM is assumed to be $15 \mathrm{~cm}^{-1}$. Prominent peaks are labeled in the observed spectrum. Very weak bands such as $\mathrm{Me}(\mathrm{C}-\mathrm{H})_{\text {asym stret }}$ at $2953.8 \mathrm{~cm}^{-1}, \operatorname{Ar}(\mathrm{C}-\mathrm{H})$ stretch at $3078.0 \mathrm{~cm}^{-1}$, etc. are not labeled in the observed spectrum. 


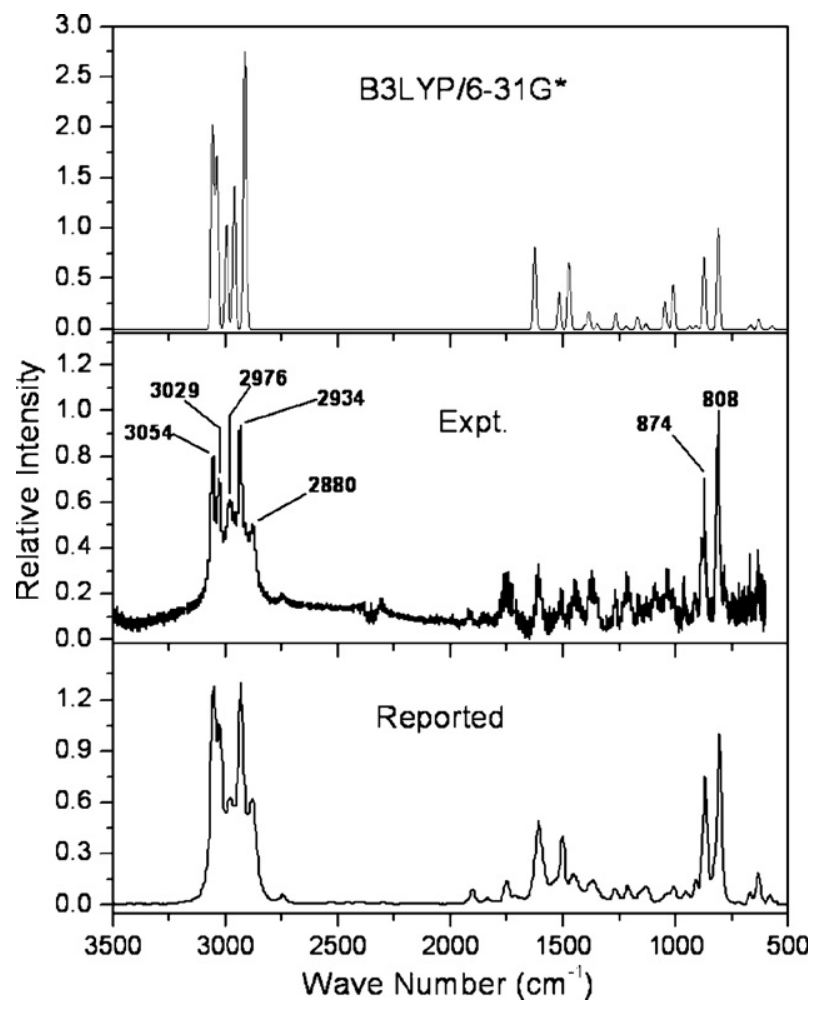

Fig. 5. Comparison of NIST reported data from [10], experimental and theoretical IR absorption spectra of 2,6-DMN. In the calculated spectrum the FWHM is assumed to be $15 \mathrm{~cm}^{-1}$. Prominent peaks are labeled in the observed spectrum. Very weak band such as $\mathrm{Me}(\mathrm{C}-\mathrm{H})_{\text {asym stret }}$ at $2955.2 \mathrm{~cm}^{-1}$, etc. are not labeled in the observed spectrum.

DMN, 747.3, 784.5, and $893.1 \mathrm{~cm}^{-1}$ in 1,6-DMN and 874.7 and $940.4 \mathrm{~cm}^{-1}$ in 2,6-DMN. The next low intensity band appears at $1034.7 \mathrm{~cm}^{-1}$ in $1,5-\mathrm{DMN}, 1038.1 \mathrm{~cm}^{-1}$ in 1,6-DMN and $1037.9 \mathrm{~cm}^{-1}$ in 2,6-DMN. This band has been assigned to a $\mathrm{Me}(\mathrm{C}-\mathrm{H})$ out-of-plane bending mode appearing at 1047.2/ $1048.5 \mathrm{~cm}^{-1}$ in $1,5-\mathrm{DMN}, 1048.2 / 1048.4$ in 1,6-DMN and $1047.6 / 1048.3 \mathrm{~cm}^{-1}$ in 2,6-DMN in calculation. $\mathrm{A} \mathrm{Me}(\mathrm{C}-\mathrm{H})$ in-plane bending vibration of low intensity is seen in 1,6-DMN at $981.9 \mathrm{~cm}^{-1}$. In 2,6-DMN the same band appears at $961.4 \mathrm{~cm}^{-1}$. The $\mathrm{Me}(\mathrm{C}-\mathrm{H})$ in-plane bending vibration is not observed in 1,5-DMN. A weak band at $1062.6 \mathrm{~cm}^{-1}$ corresponding to the Ar-ring deformation mode is seen only in 1,5-DMN which matches with the calculated band at $1069.7 \mathrm{~cm}^{-1}$.

$\operatorname{Ar}(\mathrm{C}-\mathrm{H})$ in-plane bending as well as ring deformation occurs at a slightly higher frequency. For 1,5-DMN it is observed at $1217.3 \mathrm{~cm}^{-1}$, for 1,6-DMN at $1217.5 \mathrm{~cm}^{-1}$ and for $2,6-\mathrm{DMN}$ at $1216.4 \mathrm{~cm}^{-1}$. The calculated positions, respectively, are $1258.6,1220.4$, and $1267.7 \mathrm{~cm}^{-1}$. For the first and last compounds a band at $1264.0 \mathrm{~cm}^{-1}$ and a band at $1273.0 \mathrm{~cm}^{-1}$, respectively, have been observed which do not correspond to any harmonic bands in the respective calculated spectrum. They have been tentatively assigned to either a combination or a difference band. For example, the 1264.0$\mathrm{cm}^{-1}$ band in 1,5-DMN may be a difference band arising from the two strong fundamentals $(2882.4-1606.1) \mathrm{cm}^{-1}$ while the $1273.0 \mathrm{~cm}^{-1}$ band in 2,6-DMN may be a difference band from
$(2880.0-1612.9) \mathrm{cm}^{-1}$. Another weak $\operatorname{Ar}(\mathrm{C}-\mathrm{H})$ in-plane bending occurs at $1413.9 \mathrm{~cm}^{-1}$ in $1,5-\mathrm{DMN}, 1426.5 \mathrm{~cm}^{-1}$ in 1,6-DMN, and $1365.0 \mathrm{~cm}^{-1}$ in 2,6-DMN which correspond to the calculated bands at 1416.2, 1437.9, and $1386.1 \mathrm{~cm}^{-1}$, respectively. In addition, in 1,6-DMN and 2,6-DMN a low intensity band, respectively, at 1076.0 and $1093.1 \mathrm{~cm}^{-1}$ have been assigned to $\operatorname{Ar}(\mathrm{C}-\mathrm{H})$ in-plane bending vibration. The next identifiable band in the spectra occurs at $1448.9 \mathrm{~cm}^{-1}$ in $1,5-$ DMN, $1450.0 \mathrm{~cm}^{-1}$ in 1,6-DMN, and at $1450.1 \mathrm{~cm}^{-1}$ in $2,6-$ DMN. This band corresponds to the calculated bands in the respective compounds at 1474.0/1474.1, 1473.5/1474.1, and $1472.6 / 1472.7 \mathrm{~cm}^{-1}$ belonging to the $\mathrm{Me}(\mathrm{C}-\mathrm{H})$ out-of-plane twisting. The next set of weak bands appears at 1510.9 and $1606.1 \mathrm{~cm}^{-1}$ in $1,5-\mathrm{DMN} ; 1513.6,1605.8$, and $1638.6 \mathrm{~cm}^{-1}$ in 1,6-DMN; at 1510.9 and $1612.9 \mathrm{~cm}^{-1}$ in 2,6-DMN. These bands have been identified as the $\operatorname{Ar}(\mathrm{C}-\mathrm{C})$ stretching vibrations from the calculations.

The next sets of three to four bands in the spectra of the DMNs do not correspond to any band positions in the calculated spectra and, thus, have been generally identified as combination or difference bands. In 1,5-DMN, the observed band at $1745.3 \mathrm{~cm}^{-1}$ matches with a difference band of $(2956.9-1217.3) \mathrm{cm}^{-1}$ consisting of two fundamentals and that at $1848.6 \mathrm{~cm}^{-1}$ is due to a combination of two bands $(1062.6+807.5) \mathrm{cm}^{-1}$. Similarly, a combination band at $1922.2 \mathrm{~cm}^{-1}$ of fundamental frequencies $(1062.6+872.2) \mathrm{cm}^{-1}$ has been identified. The observed band at $2744.6 \mathrm{~cm}^{-1}$ in $1,5-\mathrm{DMN}$ may be assigned as a difference band originating from the combination of fundamental frequencies $(2931.8-1217.3+1034.7) \mathrm{cm}^{-1}$. The bands observed at $1741.0,1858.9,1922.4$ and $2746.6 \mathrm{~cm}^{-1}$ in $1,6-$ DMN do not correspond to any calculated band. The band observed at $1741.0 \mathrm{~cm}^{-1}$ could be a difference band of two fundamental frequencies $(2953.8-1217.5) \mathrm{cm}^{-1}$ whereas the weak $1858.9 \mathrm{~cm}^{-1}$ band may originate from a coupled vibration of $\operatorname{Ar}(\mathrm{C}-\mathrm{H})$ in-plane bending vibration at $1076.0 \mathrm{~cm}^{-1}$ and $\operatorname{Ar}(\mathrm{C}-\mathrm{H})$ out-of-plane bending vibration at $812.8 \mathrm{~cm}^{-1}$, while the band observed at $1922.4 \mathrm{~cm}^{-1}$ is a combination band of frequencies $(1076.0+870.2) \mathrm{cm}^{-1}$. The observed band at $2746.6 \mathrm{~cm}^{-1}$ may be assigned to a difference band of fundamental frequencies (2933.3 $1217.5+1038.1) \mathrm{cm}^{-1}$. In 2,6-DMN, the observed bands at $1741.8,1908.1$ and $2744.5 \mathrm{~cm}^{-1}$ do not match with any band in the calculated spectrum. The band at $1741.8 \mathrm{~cm}^{-1}$ is likely to be a difference band of the fundamental frequencies $(2955.2-1216.4) \mathrm{cm}^{-1}$. In general, the difference and combination bands are less intense than the fundamental bands [18]. The band observed at $1908.1 \mathrm{~cm}^{-1}$ may be a combination band of frequencies $(1093.1+808.5) \mathrm{cm}^{-1}$. There is a medium intensity band observed at $2744.5 \mathrm{~cm}^{-1}$ which is a difference band of three frequencies $(2933.9-1216.4+$ 1037.9) $\mathrm{cm}^{-1}$. The next sets of bands observed in the DMNs are at high frequencies $\left(2870-3090 \mathrm{~cm}^{-1}\right)$, forms a broad envelope with several peaks and are of moderate intensities. The first three bands in this group are at 2882.4, 2931.8, and $2956.9 \mathrm{~cm}^{-1}$ in $1,5-\mathrm{DMN} ; 2874.8,2933.3$, and $2953.8 \mathrm{~cm}^{-1}$ in 1,6-DMN; 2880.0, 2933.9, and $2955.2 \mathrm{~cm}^{-1}$ in 2,6-DMN; 
Table 4

Comparison of observed $\operatorname{Ar}(\mathrm{C}-\mathrm{H})$ out-of-plane bending and methyl $\mathrm{C}-\mathrm{H}$ symmetric (a) and asymmetric stretching (b) vibrations in DMNs

\begin{tabular}{|c|c|c|c|}
\hline Mode of vibration & $1,5-\mathrm{DMN}$ & 1,6-DMN & 2,6-DMN \\
\hline $\operatorname{Ar}(\mathrm{C}-\mathrm{H})$ out-of-plane & $783.2(1.000)$ & $812.8(1.000)$ & $808.5(1.000)$ \\
\hline $\mathrm{CH}_{3}, \mathrm{C}-\mathrm{H}$ stretching & $\begin{array}{l}2882.4(0.146) \mathrm{a} \\
2931.8(0.107) \mathrm{b} \\
2956.9(0.050) \mathrm{b}\end{array}$ & $\begin{array}{l}2874.8(0.395) \mathrm{a} \\
2933.3(0.395) \mathrm{b} \\
2953.8(0.079) \mathrm{b}\end{array}$ & $\begin{array}{l}2880.0(0.212) \mathrm{a} \\
2933.9(0.549) \mathrm{b} \\
2955.2(0.012) \mathrm{b}\end{array}$ \\
\hline
\end{tabular}

The band positions are given in $\mathrm{cm}^{-1}$ and the relative intensities in parentheses.

which from comparison with calculations have been identified as the $\mathrm{Me}(\mathrm{C}-\mathrm{H})$ symmetric and asymmetric stretching fundamentals. However, the calculated bands at these frequencies are doubly degenerate for each mode due to the presence of two methyl groups in the molecules. At our experimental resolution, these degeneracies cannot be lifted. Also in calculation some of these fundamentals appear to be highly intense and have higher intensity than the low frequency $\operatorname{Ar}(\mathrm{C}-\mathrm{H})$ out-of-plane bending fundamental. In experiment, the latter remains the strongest band in the IR spectra of DMNs. The next three to four bands are also of moderate intensities and appear at 2979.4, 3043.2, and $3081.0 \mathrm{~cm}^{-1}$ in 1,5-DMN; $2980.5,3031.8,3056.8$, and $3078.0 \mathrm{~cm}^{-1}$ in 1,6-DMN; 2976.5 , 3028.9 , and $3054.5 \mathrm{~cm}^{-1}$ in $2,6-\mathrm{DMN}$. These bands correspond to three pairs of doublets in calculation as shown in Tables 1-3 and are assigned to $\operatorname{Ar}(\mathrm{C}-\mathrm{H})$ stretching modes. In 1,6-DMN the last doublet appears as the doublet in the observed spectrum and is clearly resolved.

By comparing the DMN and MN spectra, we resolve that there is only one strong band in the reported spectrum of 1-MN due to the $\operatorname{Ar}(\mathrm{C}-\mathrm{H})$ stretching vibration above $3000 \mathrm{~cm}^{-1}$ while there are two or more moderate intensity bands in the observed spectrum of the DMNs in that region. In Table 4 we have listed four characteristic bands of the DMNs, which may help in their identification in an unknown mixture. $\operatorname{The} \operatorname{Ar}(\mathrm{C}-\mathrm{H})$ out-ofplane bending vibration is the strongest in intensity among all the bands in DMNs and is easy to identify around $800 \mathrm{~cm}^{-1}$. The DMNs are distinguishable from the position of this band, which appears with a clear separation in different DMNs. Methyl C-H symmetric and asymmetric stretching set of three bands is the next set of bands, which are distinct in all the DMNs investigated in this paper. They appear clearly resolved at the high frequency end of the IR spectrum around $2900 \mathrm{~cm}^{-1}$ with moderate intensities and can be easily marked.

\section{Conclusions}

We have reported the gas phase IR spectra of 1,5-DMN, 1,6DMN and 2,6-DMN and assigned their vibrational modes using harmonic DFT calculation. In general experimental spectra are in good agreement with the calculated spectra. From the experimentally observed spectra it is possible to distinguish the DMNs based on the intensity and position of the $\operatorname{Ar}(\mathrm{C}-\mathrm{H})$ outof-plane bending and methyl $\mathrm{C}-\mathrm{H}$ symmetric and antisym- metric stretching vibrations which are well resolved in frequency among various DMNs. These spectra at $0.5 \mathrm{~cm}^{-1}$ resolution have more features over the previously reported lowresolution spectra of the DMNs. Further studies with isotope substitution along with anharmonic analysis of the data are necessary to fully assign the vibrations of these differently substituted DMNs.

\section{Acknowledgments}

The authors would like to thank K.P.J. Reddy of the Department of Aerospace Engineering for providing the FT-IR spectrometer obtained through funds from DST-FIST for this study. EA thanks Indo-French Centre for Promotion of Advanced Research (3405-3) for partial support of this research.

\section{References}

[1] F. Wania, D. Mackay, Environ. Sci. Technol. 30 (1996) 390A.

[2] P.T. Williams, K.D. Bartle, G.E. Andrews, Fuel 65 (1986) 1150.

[3] J. Bundt, W. Herbel, H. Steinhart, S. Franke, W. Francke, High Resolut. Chromatogr. 14 (1991) 91.

[4] P.F. Nelson, Fuel 68 (1989) 283.

[5] B. Zielinska, J.C. Sagebiel, G. Harshfield, A.W. Gertler, W.R. Pierson, Atmos. Environ. 30 (1996) 2269.

[6] S.J. Clemett, C.R. Maechling, R.N. Zare, P.D. Swan, R.M. Walker, Science 262 (1993) 721.

[7] J. Semmler, P.W. Yang, G.E. Crawford, Vibr. Spectrosc. 2 (1991) 189.

[8] (a) C.W. Bauschlicher Jr., J. Chem. Phys. 234 (1998) 87; (b) C.W. Bauschlicher Jr., J. Chem. Phys. 233 (1998) 29.

[9] C.W. Bauschlicher Jr., S.R. Langhoff, Chem. Phys. 234 (1998) 79.

[10] http://webbook.nist.gov/chemistry.

[11] J. Kurtz, Astron. Astrophys. 255 (1992) L1.

[12] C. Joblin, L. d'Hendecourt, A. Léger, D. Défourneau, Astron. Astrophys. 281 (1994) 923.

[13] C. Joblin, P. Boissel, A. Léger, L. d'Hendecourt, D. Défourneau, Astron. Astrophys. 299 (1995) 835.

[14] B.S. Galabov, T. Dudev, Vibrational intensities, in: J.R. Durig (Ed.), Vibrational Spectra and Structure, vol. 22, Elsevier, Amsterdam, 1996.

[15] Frisch Æleen, M.J. Frisch, GAUSSIAN 98 User's Reference, Gaussian, Inc., Pittsburgh, PA, 1999.

[16] C.W. Bauschlicher Jr., S.R. Langhoff, Spectrochim. Acta Part A 53 (1997) 1225.

[17] S.R. Langhoff, C.W. Bauschlicher Jr., D.M. Hudgins, S.A. Sandford, L.J. Allamandola, J. Phys. Chem. A 102 (1998) 1632.

[18] G. Herzberg, Infrared and Raman Spectra of Polyatomic Molecules, D. Van Nastrand Company, Inc., New York, 1946. 